Proof Schemes Combined: Mapping secondary students' multi-faceted and evolving first encounters with mathematical proof

Ioannis Kanellos, Elena Nardi ${ }^{*}$, Irene Biza

School of Education, University of East Anglia, Norwich, UK

*Corresponding author:

School of Education

University of East Anglia

Norwich NR4 7TJ

UK

e.nardi@uea.ac.uk 


\title{
Proof Schemes Combined: Mapping secondary students' multi-faceted and evolving first encounters with mathematical proof
}

\begin{abstract}
In this paper, we propose an enriched and extended application of Harel and Sowder's proof schemes taxonomy that can be used as a diagnostic tool for characterising secondary students' emergent learning of proof and proving. We illustrate this application in the analysis of data collected from 85 Year 9 (age 14 to 15) secondary students. We capture these students' first encounters with proof and proving in an educational context (mixed-ability, state schools in Greece) where mathematical proof is explicitly present in algebra and geometry lessons and where proving skills are typically expected, and rewarded, in key national examinations. We analyse student written responses to six questions, soon after the students had been introduced to proof and we identify evidence of six of the seven proof schemes proposed by Harel and Sowder as well as a further eight combinations of the six. We observed these combinations often within the response of the same student and to the same item. Here we illustrate the eight combinations and we claim that a dynamic use of the proof schemes taxonomy that encompasses sole and combined proof schemes is a potent theoretical and pedagogical tool for mapping students' multi-faceted and evolving competence in, and appreciation for, proof and proving.
\end{abstract}

Keywords: proof schemes, secondary mathematics, first encounter with proof, proof and proving

\section{Introduction}

Our focus on proof and proving is rooted in the recognition, alongside that of many other colleagues (Hanna, 1991, 2000, 2007; Hanna \& Barbeau, 2008; G.J. Stylianides \& A.J. Stylianides, 2008) of its importance in students' mathematical studies as well as of the challenges its teaching often poses (Biza, Nardi, \& Zachariades, 2009; Harel, 1999; Harel \& Sowder, 1998, 2007; Healy \& Hoyles, 2000; Heinze, 2004; Küchemann \& Hoyles, 2006; Skemp, 1976; A.J. Stylianides, 2007; A.J. Stylianides, G.J. Stylianides, \& Philippou, 2004). In this paper, we draw on the doctoral study of the first author (Kanellos, 2014) in order to explore the potency - theoretical and pedagogical - of one tool, Harel and Sowder's $(1998,2007)$ proof 
schemes taxonomy, which was put forward with the aim to enlighten understanding of students' first encounters with formal mathematical proof. We propose that an enriched and extended use of the taxonomy enhances its potency and we ground our claim on a refined analysis of data, presented first in (Kanellos, 2014). First, we embed the proof schemes taxonomy, and our choice to use it as the analytical lenses for our data, into the broader literature on proof and proving. We then introduce the context, aims and methods of the study. The main part of the paper comprises illustrations of our enriched and extended use of the proof schemes taxonomy as deployed in the analysis of responses to six proof questions by 85 Year 9 students. We conclude with a brief discussion of the potentialities for theory and pedagogy that our proposed use of proof schemes implies.

\section{Research into students' first encounters with proof and proving}

Students' introduction to proof has been extensively studied at post-secondary level (Hanna, 2000, 2002, 2007; Hanna \& Barbeau, 2008; Harel \& Sowder, 1998, 2007; Weber \& Alcock, 2004), widely at secondary (e.g. Healy \& Hoyles, 2000; Küchemann \& Hoyles, 2006) and less widely at primary level (e.g. A.J. Stylianides, 2007). In this study, we are interested in those works that discuss proof and proving at school level, where, depending on the curricular priorities of the educational contexts in which studies are conducted, proof has varying degrees of presence. Within a primary school context, A.J. Stylianides (2007), for example, introduces a definition of proof for primary school students that serves the needs of students, resonates with the norms of mathematics as a discipline and is coherently conceptualised through the different educational levels. Within a secondary school context, Healy and Hoyles (2000) discuss students' perspectives on proof and they identify two types of arguments: those which satisfy the teacher and receive the best mark (e.g. algebraic arguments) and those which convince the students personally (empirical arguments). The students in their study seem to 
think mostly empirically when it comes to proof and problem solving, although most of them are aware of the limitations of an empirical approach.

As we explain in detail in the next section, in the educational context of our study - Year 9, Greek compulsory education - proof is explicitly present in the textbook (Argyrakis, Vourganas, Mentis, Tsikopoulou \& Chryssovergis, 2010), lessons and assessment. Although a formal introduction to deductive proof comes later in the curriculum, there is an expectation from students to prove simple statements in algebra and geometry. This is seen as a step towards their enculturation to deductive proof approaches. In our study, we investigate secondary students' first encounters with formal mathematical proof. To this aim, we have been in search of a diagnostic tool that can examine these early encounters, particularly in the light of what is seen within the discipline of mathematics as acceptable deductive proof. Our analysis aims to capture the students' transition from informal, empirical approaches to deductive reasoning, extending and strengthening prior reports of this transition (e.g. Küchemann \& Hoyles, 2006).

One example of a construct that appeared to us in the earlier stages of our study as having this diagnostic capacity is Balacheff's (1987) distinction between pragmatic proofs (preuves pragmatiques) and conceptual proofs (preuves intellectuelles). Balacheff identified various approaches to proving: (a) nä̈ve empiricism (empirisme naïf) consists of concluding the truth of an assertion from the observation of a small number of cases; (b) crucial experiment (expérience cruciale) is a validation process of an assertion where the individual explicitly poses the problem of generalisation and resolves it, betting on a case in which s/he recognises the less particular as possible; (c) generic example (exemple générique) involves making explicit the reasons for the validity of an assertion by means of the realisation of operations or transformations of an object that is not present itself but is a characteristic representative of a class of individuals; and, (d) mental experiment (expérience mentale) involves interiorising and 
detaching an action from its realisation by a particular representative. Naïve empiricism, crucial experiment and generic example are seen as pragmatic proofs while mental experiments are seen as conceptual proofs.

An even more elaborate distinction between variations of empirical (mostly inductive) and deductive proof is also fundamental in the proof schemes taxonomy suggested by Harel and Sowder (1998, 2007). Proof schemes (1998) is a framework for students' perceptions of proof, grounded on observations and teaching experiments with students in tertiary education in three mathematical areas: geometry, linear algebra, and elementary number theory. Later, Harel and Sowder (2007) drew on a range of factors - cognitive, mathematical, historicalepistemological and institutional-sociocultural - to propose an elaborated taxonomy of the proof schemes and its characteristics. As the more elaborate version of the taxonomy is the one that we deploy in the analyses presented in this paper, we present this briefly below.

According to Harel and Sowder (2007), proof schemes describe what "constitutes ascertaining and persuading" (p. 809) in the proving activity of individuals or communities, with "ascertaining" being the removal of their own doubts and "persuading" being the removal of the doubts of others. The proof schemes evidenced in the proving activity of the students in their studies were grouped in three classes: external conviction proof schemes, empirical proof schemes, and deductive proof schemes, with each divided further in subclasses.

External conviction proof schemes are grounded on external to the learner sources of conviction and are classified in: (a) authoritarian proof schemes, if an authority such as a teacher or a book is appealed to in support of an argument; (b) ritual proof schemes, if the argument depends strictly on its "appearance [...] (for example, proofs in geometry must have a two-column format)" (p. 809); and (c) non-referential symbolic proof schemes, if the argument depends "on symbol manipulations, with the symbols or the manipulations having no potential coherent system of referents (e.g., quantitative, spatial, etc.) in the eyes of the 
student (e.g., $\frac{a+b}{c+b}=\frac{a+b}{c+b}=\frac{a}{c}$ )" (ibid, p. 809).

Empirical proof schemes are grounded on experience which is deployed as evidence in the proving process and are classified in: (a) inductive proof schemes, if they rely on "evidence from examples (sometimes just one example) or direct measurements of quantities, substitutions of specific numbers in algebraic expressions" (p. 809) and (b) perceptual proof schemes, if they rely on "perceptions" (p. 809), for example, of properties of a particular function as these properties appear on a graph.

Deductive proof schemes are grounded on deductive reasoning and share the three characteristics of generality, operational thought and logical inference (ibid.). Arguments in these schemes are intended as valid for all cases and not just for single or a few cases (generality), are organised in appropriate steps to reach the final goal that completes the proof (operational thought) and are based on logical rules (logical inference). Deductive proof schemes are classified in: (a) transformational proof schemes that share the above characteristics and (b) axiomatic proof schemes which also include the acknowledgement of the axiomatic foundation of the corresponding mathematical theory, namely starting from accepted principles and building propositions through logical arguments grounded on those principles and other, already built propositions. Harel (2007) drew on historical and philosophical observations to refine the essential differences between these two types of deductive proof schemes by offering additional characteristics. Here we do not elaborate these characteristics as we see axiomatic proof schemes of limited relevance to the first - and typically not connected to sense-making of the axiomatic foundations of mathematical theory - encounters with proof of the secondary students which are the focus of this paper. We summarise the taxonomy of the proof schemes and the notation we have used in our analysis in Table 1. 
Table 1. The proof schemes taxonomy and the coding notation used in the analysis.

\begin{tabular}{lc}
\hline Proof Schemes & Codes \\
\hline External conviction proof schemes & EC. \\
Ritual proof schemes & EC.R. \\
Authoritarian proof schemes & EC.A. \\
Non-referential symbolic proof schemes & EC.NRS. \\
Empirical proof schemes & E. \\
Inductive proof schemes & E.I. \\
Perceptual proof schemes & E.P. \\
Deductive proof schemes & D. \\
Transformational proof schemes & D.T. \\
Axiomatic proof schemes & D.A. \\
\hline
\end{tabular}

We trialled Balacheff's (1987) and Harel and Sowder's (2007) taxonomies in a pilot phase of the study (Kanellos, 2014) on data collected from secondary school students' responses to proof questions from several classes and across Years 9 and 10. Both classifications captured the distinction between the empirical and the deductive proof we expected to identify in students' responses. However, the proof schemes taxonomy appeared to us to be a better fit to our purpose in the following senses: (i) it considers institutional factors such as teacher or book authority; (ii) it captures other activity such as the "ritualised" following by a student of a proof structure proposed by the book or the teacher; and, (iii) it captures incoherencies and inconsistencies in the use of symbols. Also, the proof schemes taxonomy shifts the focus from the epistemic nature of proof onto students' proving activity, while maintaining a sharp focus on the proving norms that are acceptable within the discipline of mathematics.

Given that the proof schemes taxonomy emerged initially in a post-secondary context (Harel and Sowder, 1998), we were apprehensive about the appropriateness of our choice. We were encouraged though by some other uses at secondary level that we identified in the literature. Csíkos (1999) used the initial proof scheme taxonomy (Harel and Sowder, 1998) to 
measure proving ability in mathematics, science and everyday claims of 2572 students (age 11 to 17) and identified a relationship between proof types used in different domains and school grades in mathematics. Another example of a use of the taxonomy in a secondary context is the study of Lee (2016) which traces transformational proof schemes in the responses of secondary school students in proof tasks. Lee draws on the taxonomy, together with other taxonomies (e.g. Balacheff's), to analyse the responses of 60 Singaporean secondary students (age 14 to 15 ) to deductive-proof and proof-by-counterexample tasks in elementary number theory and quadratic functions. The analysis suggests seven hierarchical levels of deductiveproof production, ranging from irrelevant inferences to deductive proofs using formal representations and six hierarchical levels of proof-by-counterexample production ranging from irrelevant inferences to construction of a general set of counterexamples.

Central to the proposition we put forward in this paper, is the observation in previous studies that evidence of different proof schemes may be found within the proving activity by a single student. Housman and Porter's (2003) outcomes from a study of post-secondary students, for example, indicate that there may exist evidence of different proof schemes in the responses to different questions by the same student. Lee (2016) presented analysis which identifies different behaviours appearing simultaneously in the response to the same question by the same participant (such as attempts at deductive inference co-existing with informal proof constructions in the same response). This is a conjecture that we investigate further.

In resonance with our study objectives - the investigation of secondary students' emergent learning of proof and proving in relation to deductive proof production - and the literature we briefly review here, we examine whether, and if so how, the analytical lenses of the Harel and Sowder (2007) taxonomy can be operationalised towards characterising secondary students' first encounter with proof and proving. The research question we therefore investigate in this paper is the following: 
RQ: Can the Harel and Sowder proof schemes taxonomy be deployed in the characterization of secondary students' first encounter with proof and proving? If so, how?

To answer this research question, we sample from the analysis of written responses to proving tasks collected from four classes of 85 Year 9 students. In a nutshell, this analysis the entirety of which is in (Kanellos, 2014) - revealed evidence of these secondary students' preparedness and appreciation for proof and proving and a willingness to engage with deductive proof production, even at these early stages of their encounter with proof and even when their technical fluency in doing so was still understandably not fully developed (Kanellos, Nardi \& Biza, 2013). This preparedness was identified in our data sometimes alongside the reliance on empirical and external conviction proof schemes that is typically reported in studies of secondary students' encounters with proof. This cohabitation of a range of dispositions towards proof was an intriguing feature of the students' responses and we credit the identification of this element to the nuance allowed by the proof schemes taxonomy - and particularly by the application of the enriched extension of the taxonomy that we present in this paper.

In the next section, we introduce the context, participants and data collection of the study and then proceed with sampling from our data analysis in order to illustrate the potentialities of an enriched and extended use of the proof schemes taxonomy - and thus provide an affirmative, and elaborate, answer to our RQ.

\section{The context, participants and data collection of the study}

The study we report in this paper was conducted in Greece, where students begin to be taught proof in algebra and in geometry in Year 9 (age 14 to 15). In algebra, they learn to prove identities such as $(\boldsymbol{a}+\boldsymbol{b})(\boldsymbol{a}-\boldsymbol{b})=\boldsymbol{a}^{\mathbf{2}}-\boldsymbol{b}^{\mathbf{2}}$ or implications such as, "if $\boldsymbol{x}<-\mathbf{2}$ then $-\mathbf{3} \boldsymbol{x}+$ 
$7>13^{\prime}$. In geometry, they learn to prove propositions based mainly on the congruency criteria of triangles. For example, they learn how to prove that every point of the bisector of an angle is equidistant from the sides of the angle (Argyrakis, et al., 2010). Thus, Year 9 is a turning point for the students, since empirical verification of mathematical statements leads gradually to logical reasoning and structured argumentation based on definitions and properties of mathematical objects.

Our study takes a snapshot of the teaching and learning of proof during Year 9. To this end, we collaborated with the teachers and students of a mixed ability, state-funded, Greek secondary school. We worked more closely with one of the teachers and her four Year 9 classes of, in total, 85 students, in algebra and geometry. We carried out extensive observations of her teaching and collaborated on the construction of the tests she administered to the students throughout the year.

Our data collection included two questionnaires administered in the beginning and at the end of the school year, with a combination of algebra and geometry items. In this paper, we analyse data from the end of year questionnaire (Table 2). 
Table 2. The proof questionnaire (translation from Greek).

Q1 For real numbers $a$ and $b$ it is given that $a^{2}+b^{2}=5^{2}$. Prove that $(a \sqrt{3}+b \sqrt{2})^{2}+$ $(a \sqrt{2}-b \sqrt{3})^{2}=125$.

$\mathrm{Q} 2 \mathrm{a} / \mathrm{b} \quad$ If the difference of the squares of two natural numbers $\kappa$ and $\lambda(\kappa>\lambda)$ is equal to the sum of these numbers then:

a. Prove that the difference of these numbers is equal to one.

b. Prove that $5556^{2}-5555^{2}=11111$.

Q3a/b Two of your peers wonder how to prove that $(\boldsymbol{a}+\boldsymbol{b})(\boldsymbol{a}-\boldsymbol{b})=\boldsymbol{a}^{\mathbf{2}}-\boldsymbol{b}^{\mathbf{2}}$. One of them suggests they should assign numerical values to $a$ and $b$ (e.g. $a=2$ and $b=1$ ), then compute both sides and check whether the right part is equal to the left part. They experiment with a number of values and they convince themselves that the result is the same for the left part as well for the right one.

a. Do you agree with your peers? If you do not agree, what would you suggest to them?

b. Do you believe that their teacher would agree with them?

Q4a/b A non-rectangular parallelogram АВГ $\Delta$ is given. From the vertex $\mathrm{A}$ we draw a perpendicular line $(\alpha)$ to $\Delta \Gamma$. Line $(\alpha)$ intersects line $\Delta \Gamma$ at point $E$.

From vertex $\Gamma$ we draw line $(\beta)$ perpendicular to line $\mathrm{AB}$. Line $(\beta)$ intersects the side

$\mathrm{AB}$ at point $\mathrm{Z}$.

a. Draw the figure.

b. Prove that triangle $\mathrm{A} \triangle \mathrm{E}$ is equal to triangle $\Gamma \mathrm{BZ}$.

Q5 In the figure below the following are valid: Line $\zeta$ passing through points $A$ and $B$ is the perpendicular bisector of the line segment $\Gamma \Delta$. Prove that the triangles $A B \Gamma$ and $\mathrm{AB} \Delta$ are congruent.

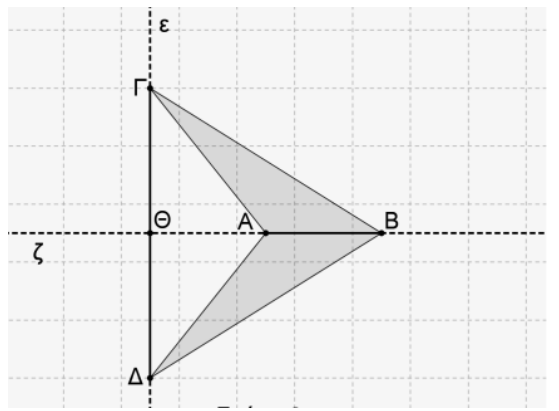

Q6a/b In the figure below triangles $\mathrm{A} \Gamma \mathrm{B}$ and $\mathrm{EB} \Delta$ have $\mathrm{A} \Gamma=\mathrm{EB}, \mathrm{AB}=\mathrm{E} \Delta$ and $\Gamma \mathrm{B}=\mathrm{B} \Delta$. Points

$\mathrm{A}, \mathrm{B}$ and $\Delta$ lie on the same line $(\alpha)$.

a. Prove that triangles $\mathrm{A} \Gamma \mathrm{B}$ and $\mathrm{EB} \Delta$ are equal.

b. Prove that lines $\mathrm{B} \Gamma$ and $\mathrm{E} \Delta$ are parallel.

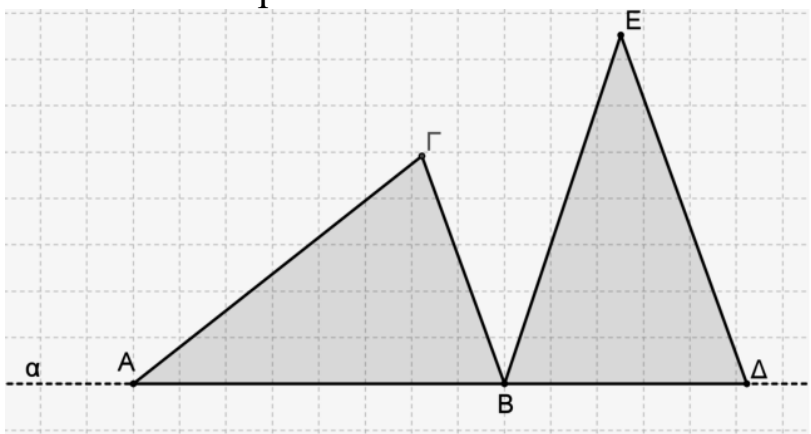


The questionnaire items were either similar to those that students had practised across the year in algebra (Q1 and Q2a,b) and geometry lessons (Q4a,b; Q5 and Q6a,b) or adjusted from items found in the literature on proof and proving (e.g. Q3 is inspired by a similar item in Healy \& Hoyles, 2000). Q1, Q2, Q4-6 measure primarily proof fluency, whereas Q3 measures primarily proof appreciation. We note that Q1, Q2, Q4-6 are relatively demanding for students at this early stage of their encounter with proving tasks. We did that on purpose: we did not want to give tasks that yielded an overoptimistic image of the students' proof fluency. We aimed to give tasks that, although familiar to the students, somewhat challenged them and invited them to deploy their emerging proving skills, while also engaging with substantial manipulation of mathematical notation. We thus consciously took the risk of a high number of scripts without solutions. We note that students were allowed ample time for producing their responses. We also note that the exemplification of our analysis of student responses covers all the questions except $\mathrm{Q} 4 \mathrm{a}$ and $\mathrm{Q} 2 \mathrm{~b}$, since we did not generate proof-scheme characterisations for the responses to these two: the former invites the students to draw a figure and the latter requires a numerical calculation only.

In order to analyse the student scripts, we constructed a spreadsheet - the entirety of which is in (Kanellos, 2014) and an excerpt corresponding to the entries for the participants we discuss in this paper is in Table 4 - with the following structure. Rows correspond to participants and columns correspond to questions (or sub-questions, for questions such as Q6 which consist of more than one part). The content of each cell is an abbreviation of the proof scheme we thought the proof of the respective participant provided evidence of (we used the coding in Table 1). In this sense, every row provides the proof scheme evident in the responses of a particular participant; and, every column provides the evidence of certain proof schemes in the students' responses to a particular question. In the cases where we identified evidence of more than one proof scheme in a response, more than one code was added in the respective cell 
(e.g. participant 09 in Q6a and Q6b in Table 4). A sample of the responses were analysed by each author individually and discussed until consensus was reached. Then, the first author analysed the rest of the responses and only the cases he found ambiguous were discussed by the whole team.

The analysis identified evidence of six of the seven proof schemes proposed by Harel and Sowder: as we noted earlier, there was no evidence of D.A. (deductive - axiomatic) proof schemes in the student responses. This is not surprising given that the students encounter proof in Year 9 not as part of an introduction to the axiomatic foundations of, say, Euclidean Geometry. This introduction occurs in Year 10. During the analysis, we also observed the high occurrence of cells in the spreadsheet where more than one proof scheme code had been entered. Specifically, there were these eight combinations of different proof schemes (Table 3).

Table 3. Eight combinations of proof schemes identified in the student responses.

\begin{tabular}{|c|c|}
\hline Code & Proof scheme combination \\
\hline D.T.-E.I. & Deductive Transformational - Empirical Inductive \\
\hline D.T.-E.P. & Deductive Transformational - Empirical Perceptual \\
\hline D.T.-EC.A. & Deductive Transformational - External Conviction Authoritarian \\
\hline D.T.-EC.NRS. & $\begin{array}{l}\text { Deductive Transformational - External Conviction Non-Referential } \\
\text { Symbolic }\end{array}$ \\
\hline E.I.-EC.NRS. & Empirical Inductive - External Conviction Non-Referential Symbolic \\
\hline E.P.-EC.A. & Empirical Perceptual - External Conviction Authoritarian \\
\hline E.P.-EC.NRS. & Empirical Perceptual - External Conviction Non-Referential Symbolic \\
\hline EC.R.-EC.NRS. & $\begin{array}{l}\text { External Conviction Ritual - External Conviction Non-referential } \\
\text { symbolic }\end{array}$ \\
\hline
\end{tabular}

Naturally, some of the combinations occurred with higher frequency than others in the student scripts. In our exemplification from the eight combinations that follows in the next section, we comment on this occasionally but we also stress that the frequencies of the various, sole and combined, proof schemes - and what these imply for the students' emerging proof competence, confidence and appreciation - are discussed in (Kanellos, 2014) and that this 
discussion is not the focus of this paper. Our aim in this paper is to illustrate the eight combinations through exemplification from our data and thus to propose the theoretical and pedagogical potency of this enriched and extended application of the proof schemes taxonomy.

\section{Data Analysis}

Evidence of six proof schemes proposed by Harel and Sowder (2007) were identified in the students' responses either sole or in combinations. We now present briefly examples of students' responses in which we identified evidence of one proof scheme only (sole proofscheme responses, 4.1). We then dedicate the bulk of this section (4.2) to exemplifying from student responses in which we identified evidence of combinations of proof schemes.

Table 4. Proof Scheme characterisations: Participant $(\mathrm{P})$ scripts exemplified in this paper.

\begin{tabular}{|c|c|c|c|c|c|c|c|c|}
\hline $\mathrm{P}$ & Q1 & Q2a & Q3a & Q3b & Q4b & Q5 & Q6a & Q6b \\
\hline 03 & D.T. & D.T. & D.T. & D.T. & D.T. & D.T. & E.P. & E.P. \\
\hline 09 & D.T. & E.I. & EC.A. & EC.A. & EC.NRS. & EC.NRS. & $\begin{array}{c}\text { E.P.- } \\
\text { EC.NRS. }\end{array}$ & $\begin{array}{c}\text { E.P.- } \\
\text { EC.NRS. }\end{array}$ \\
\hline 11 & E.I. & $\begin{array}{c}\text { E.I.- } \\
\text { EC.NRS. }\end{array}$ & D.T. & D.T. & $\begin{array}{c}\text { E.P.- } \\
\text { EC.NRS. }\end{array}$ & $\begin{array}{c}\text { E.P.- } \\
\text { EC.NRS. }\end{array}$ & D.T. & $\begin{array}{c}\text { E.P.- } \\
\text { EC.NRS. }\end{array}$ \\
\hline 32 & EC.NRS. & EC.NRS. & D.T. & D.T. & $\begin{array}{c}\text { EC.R.- } \\
\text { EC.NRS. }\end{array}$ & EC.NRS. & D.T. & $\begin{array}{c}\text { E.P.- } \\
\text { EC.NRS. }\end{array}$ \\
\hline 37 & D.T.-E.I. & D.T. & D.T. & D.T. & $\begin{array}{c}\text { D.T.- } \\
\text { EC.NRS. }\end{array}$ & D.T. & D.T. & D.T. \\
\hline 39 & N.S. & E.I. & N.S. & N.S. & $\begin{array}{c}\text { EC.NRS.- } \\
\text { EC.R. }\end{array}$ & N.S. & N.S. & N.S. \\
\hline 51 & N.S. & N.S. & E.I. & E.I. & EC.NRS. & $\begin{array}{l}\text { E.P.- } \\
\text { EC.A. }\end{array}$ & D.T. & EC.NRS. \\
\hline 52 & $\begin{array}{c}\text { E.I.- } \\
\text { EC.NRS. }\end{array}$ & N.S. & D.T. & D.T. & N.S. & EC.NRS. & $\begin{array}{c}\text { D.T.- } \\
\text { EC.NRS. }\end{array}$ & N.S. \\
\hline 60 & N.S. & E.I. & D.T. & D.T. & $\begin{array}{c}\text { D.T.- } \\
\text { EC.NRS. }\end{array}$ & D.T. & D.T. & $\begin{array}{c}\text { E.P.- } \\
\text { EC.NRS. }\end{array}$ \\
\hline 65 & N.S. & N.S. & D.T.-E.I. & $\begin{array}{l}\text { D.T.- } \\
\text { EC.A. }\end{array}$ & E.P. & $\begin{array}{l}\text { D.T.- } \\
\text { E.P. }\end{array}$ & D.T. & N.S. \\
\hline 68 & $\begin{array}{c}\text { D.T.- } \\
\text { EC.NRS. }\end{array}$ & N.S. & D.T.-E.I. & D.T. & D.T. & $\begin{array}{l}\text { D.T.- } \\
\text { E.P. }\end{array}$ & D.T. & N.S. \\
\hline 72 & EC.NRS. & $\begin{array}{c}\text { E.I.- } \\
\text { EC.NRS. }\end{array}$ & N.S. & N.S. & D.T.-E.P. & N.S. & N.S. & EC.NRS. \\
\hline 81 & D.T. & EC.NRS. & D.T.-E.I. & EC.A. & D.T. & $\begin{array}{l}\text { D.T.- } \\
\text { E.P. }\end{array}$ & D.T. & D.T. \\
\hline 85 & $\begin{array}{c}\text { D.T.- } \\
\text { EC.NRS. }\end{array}$ & $\begin{array}{c}\text { D.T.- } \\
\text { EC.NRS. }\end{array}$ & $\begin{array}{c}\text { D.T.- } \\
\text { EC.NRS. }\end{array}$ & $\begin{array}{c}\text { D.T.- } \\
\text { EC.NRS. }\end{array}$ & EC.NRS. & $\begin{array}{c}\text { D.T.- } \\
\text { EC.NRS. }\end{array}$ & D.T. & N.S. \\
\hline
\end{tabular}




\subsection{Sole proof schemes}

All responses classified as including evidence of EC.A. (External Conviction Authoritarian) proof schemes were responses to Q3. Participant 09 (thereafter ${ }^{1} \mathrm{P}[09]$ ) responded to Q3a:

" $a^{2}-b^{2}$ is factorised as follows $(a+b)(a-b)$ and also is an identity and the law of identities says that the result is valid for whichever values it takes"

And to Q3b:

"No the teacher would not agree he would say to them the above and to open their books".

In the first part of the question, the student takes the algebraic identity $a^{2}-b^{2}=(a+b)(a-$ $b$ ) as given, and not as an equality to be proved. He does not therefore feel the need to prove it. In the second part of the question, he says that the teacher will ask the students to check the list of algebraic identities in the book. In this response, the book and the given list of algebraic identities is the authority that ascertains $\mathrm{P}[09]$ and to which the teacher is expected to resort. For this reason, we characterised this response as EC.A..

Evidence of the EC.NRS. (External Conviction Non-Referential Symbolic) proof scheme was identified in student responses across all items of the questionnaire. For example, in Figure 1 we present P[72]'s response to Q1 where " $(\alpha-5)(\alpha+5)$ ” is written as " $\alpha-5(\alpha+5)$ " and then " $\beta^{2}$ $4 \alpha-25=0$ " is meant to be solved as a quadratic equation $\left(a x^{2}+b x+c=0\right)$ with the use of the formula $\Delta=b^{2}-4 a c$. The incoherency of the symbolic manipulation in this response led us to the characterisation EC.NRS..

\footnotetext{
${ }^{1}$ We name the participants $\mathrm{P}[\mathrm{X}]$, where $\mathrm{X}$ is the student number we used to anonymise the student scripts.
} 


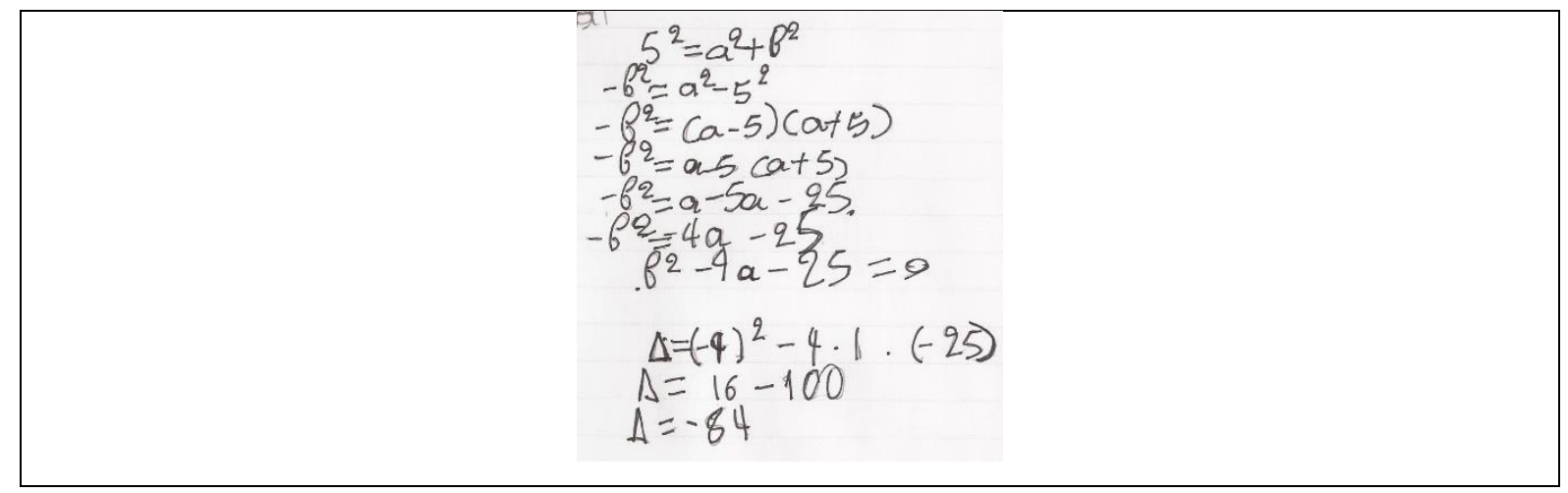

Figure 1. P[72]'s response to Q1.

There were responses with evidence of EC.R. (External Conviction-Ritual) proof schemes which, because they consisted only of listing the information given in the task and no further processing of this information by the student, were characterised, also, as "nonsolutions". For example, several students draw on paper an arrangement for their proof that was often used in class: a two column (or row) set-up entitled "hypothesis" and "conclusion" and potentially a third column (or row) for "proof". Then, they leave this blank (see, for example, P[39]'s attempt at Q6 in Figure 2). These students seem to be aware of, and willing to follow, what they perceive as the teacher's normal expectation of what a proof looks like, but do not proceed further with placing anything within each column. We note that there exist accomplished responses that follow this three-section format too (we cite one of these later, $\mathrm{P}[81]$ 's response to Q6 in Figure 5).

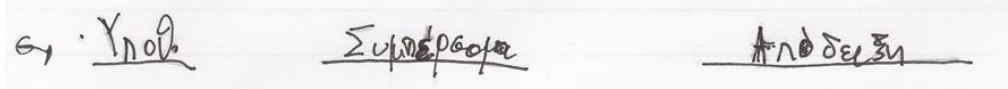

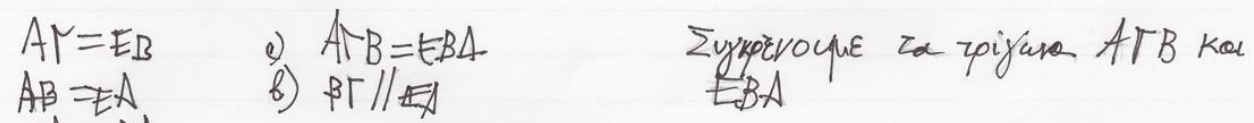

$$
\begin{aligned}
& \Gamma A=A A
\end{aligned}
$$

Figure 2. P[39]'s response to Q6. Headings: "Hypothesis", "Conclusion", "Proof". Text in third column: "We compare triangles $\mathrm{A} \Gamma \mathrm{B}$ and $\mathrm{EB} \Delta$ " [translated from Greek].

Evidence of Empirical Inductive (E.I.) proof schemes was identified in the student responses mostly in the algebra items. For example, in her attempt at Q2a (Figure 3), P[60] has chosen two specific natural numbers with difference one, and shows that the given equation is satisfied. $\mathrm{P}[60]$ 's whole argument is based on a specific pair of natural numbers to verify the 
inverse inference, namely that: if a difference of two numbers is one, then the difference of their squares is equal to the sum of these numbers. For this reason, we characterised this as E.I..

\begin{tabular}{|c|c|c|c|}
\hline $\begin{array}{l}(k-\lambda)(k+\lambda)=k+\lambda \\
(2-3)(2+1)=2+1 \\
m+3=3 \\
3=3 \quad \text { op } 80^{\prime}\end{array}$ & E6r20 $\begin{aligned} k & =2 \\
\lambda & =1\end{aligned}$ & $\begin{array}{l}(\kappa-\lambda)(\kappa+\lambda)=\kappa+\lambda \\
(2-1)(2+1)=2+1 \\
\text { [strikethrough] } \\
13=3 \\
3=3 \text { correct }\end{array}$ & $\begin{array}{l}\text { Let's } \kappa=2 \\
\text { [and] } \lambda=1\end{array}$ \\
\hline
\end{tabular}

Figure 3a. P[60]'s response to Q2a.

Figure 3b. P[60]'s translated response to Q2a.

As we expected, exclusively E.P. (Empirical Perceptual) responses were identified mostly in the geometry items. For example, P[03]'s response to Q6a (Figure 4) is based on the perception of the triangles as isosceles, a property that was not given in the problem and was derived by the student from the image. On these grounds we characterised this response as E.P..

\begin{tabular}{|c|c|}
\hline 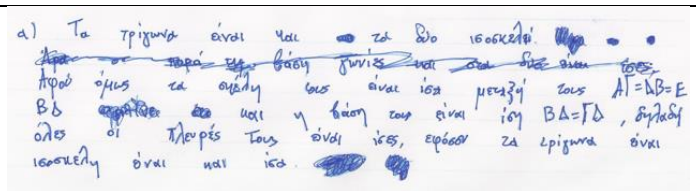 & $\begin{array}{l}\text { The triangles are both isosceles. } \\
\text { Thus the angles at the base are equal. } \\
\text { But since the equal sides of each are congruent to each other } \mathrm{A} \Gamma=\mathrm{AB}=\mathrm{E}[\Delta] \\
\mathrm{B} \Delta \text { [sic] [strikethrough] and their bases are congruent } \mathrm{B} \Delta=\Gamma \Delta \text { namely } \\
\text { all their sides are congruent since the triangles are } \\
\text { isosceles they are congruent. }\end{array}$ \\
\hline
\end{tabular}

Figure 4a. P[03]'s response to Q6a.

Figure 4b. P[03]'s translated response to Q6a.

Finally, an example that we classified as a D.T. (Deductive-Transformational) proof scheme response is from $\mathrm{P}[81]$ to $\mathrm{Q} 6$ (Figure 5). $\mathrm{P}[81$ ] reproduces the figure and states the "Hypothesis" and the "Conclusion" of the problem. Then, under the "Proof" heading, she compares the triangles by using the congruence criteria and the given information of Q6a; and, she uses the equal angles conclusion she has derived from the triangle congruency to justify why $\mathrm{B} \Gamma$ and $\mathrm{E} \Delta$ are parallel (Q6b). All the theorems she has used about congruent triangles and corresponding angle had been taught in the lessons at the time data collection took place. 


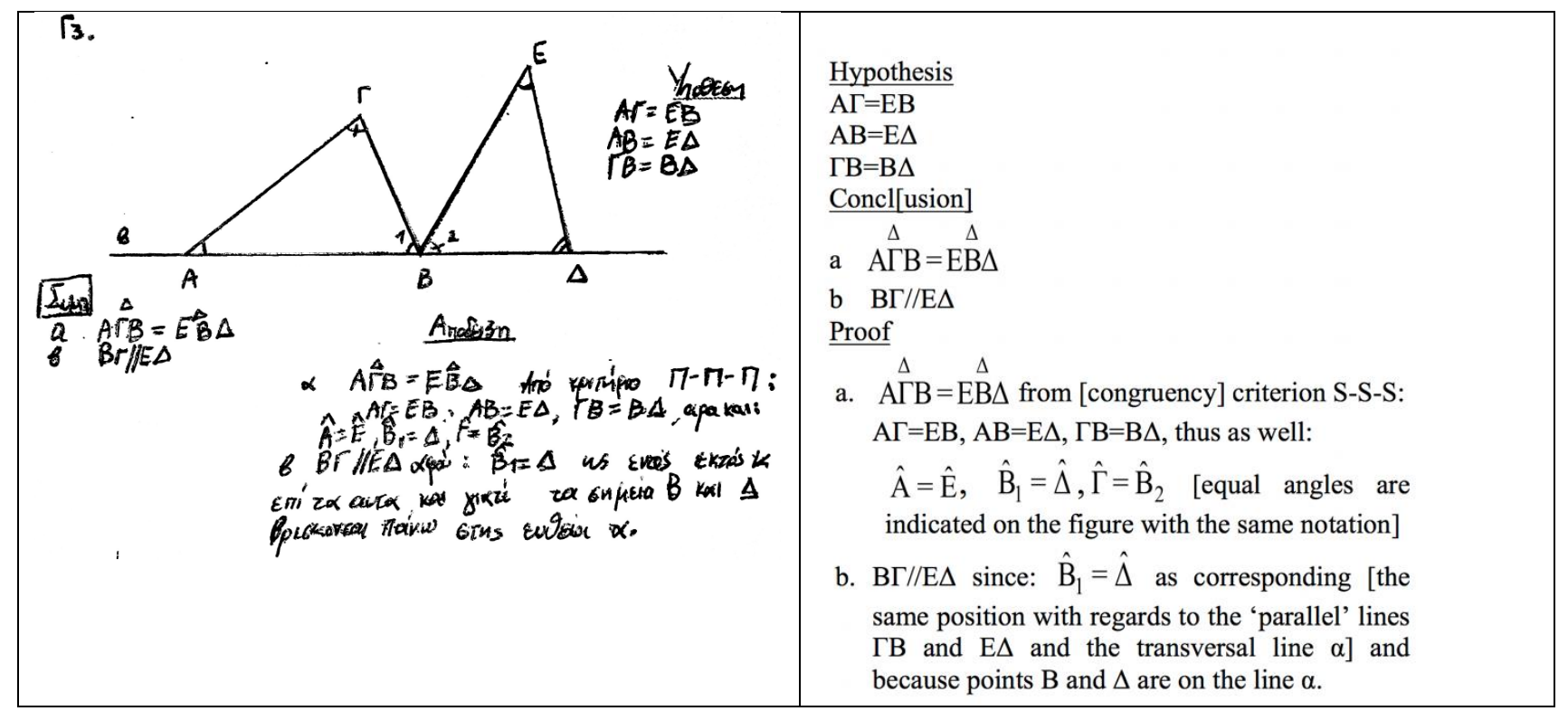

Figure 5a. P[81]'s response to Q6.

Figure 5b. P[81]'s translated response to Q6.

We now provide examples of student responses in which we identified evidence of the eight proof scheme combinations listed in Table 3.

\subsection{Combined proof schemes}

Deductive Transformational and Empirical Inductive proof schemes (D.T. - E.I.)

The first example we present is from P[37]'s response to Q1 (Figure 6). His response has two parts presented one below the other in the script. In the first part of his response (Figure 6a), he starts with the algebraic expression to be proved and follows a series of precise algebraic manipulations that lead to the equality $\alpha^{2}+\beta^{2}=5^{2}$. Then, in the second part, which follows exactly after the first part (Figure 6b), he writes "Because of the Pythagorean triad", chooses the values 3 and 4 for $\alpha$ and $\beta$ (or $\beta$ and $\alpha$ ) and verifies the equality $\alpha^{2}+\beta^{2}=5^{2}$. In the first part, algebraic notation is used with accurate application of algebraic identities in a series of expressions that involve the manipulation of square roots and $\alpha$ and $\beta . \mathrm{P}[37]$ has chosen to start from the equality to be proved and work backwards and towards $\alpha^{2}+\beta^{2}=5^{2}-$ instead of starting from the left-hand side of the equation and concluding to the right-hand side. Each step follows the preceding one with the logical symbol of implication, “ $\Rightarrow$ ", which is used correctly in his 
response. However, instead of the requested statement $" \mathrm{a} \mathrm{a}^{2}+\mathrm{b}^{2}=5^{2} \Rightarrow$ $(a \sqrt{3}+b \sqrt{2})^{2}+(a \sqrt{2}-b \sqrt{3})^{2}=125$ ", he proves the opposite statement:

“ $(a \sqrt{3}+b \sqrt{2})^{2}+(a \sqrt{2}-b \sqrt{3})^{2}=125 \Rightarrow a^{2}+b^{2}=5^{2}$ ". Despite this flaw in the logical direction of the response, we could see a commendable attempt at generality, operational thought and logical inference. We thus characterised this response as D.T..

In the second part of his response though, $\mathrm{P}[37]$ has chosen specific pairs of numbers that satisfy the equality $\alpha^{2}+\beta^{2}=5^{2}$ (there are infinite pairs of real numbers that satisfy this equality). This is not an unreasonable choice for a Year 9 student who is familiar and has practised with Pythagorean triads (in this case 3,4,5). It may be the case that $\mathrm{P}[37]$ here simply resorts to a well-known habit of students under test duress to demonstrate all that he knows about these numbers. However, the use of specific numbers as part of his response and the use of "Because" in his writing may also indicate uncertainty about the sufficiency of the algebraic proof which he may feel can be strengthened by offering a plausible example that satisfies the given equality. For this reason, we characterised this part of P[37]'s response as E.I.. Combined with the earlier characterisation of D.T., P[37]'s response to Q1 was characterised as D.T. - E.I..

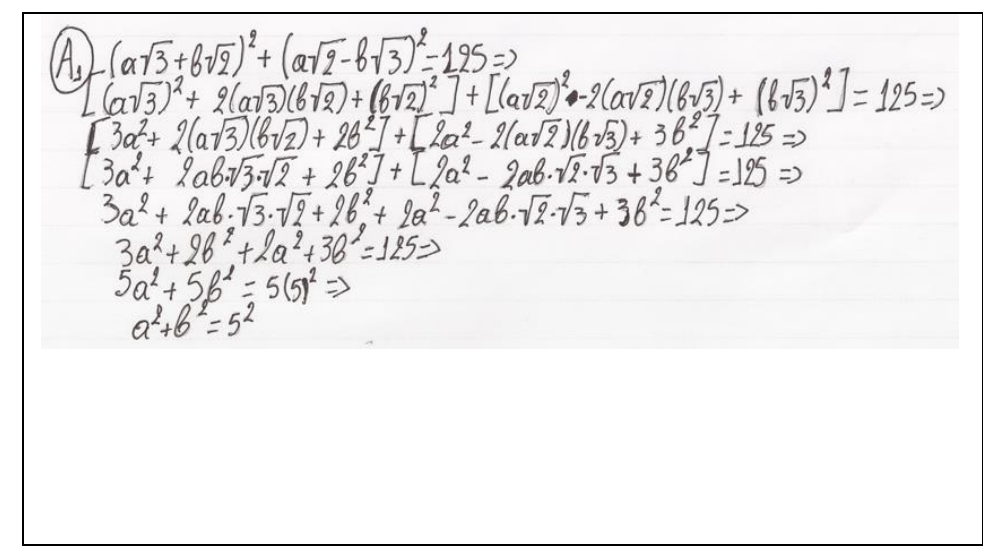

Figure 6a. P[37]'s response to Q1, Part I.

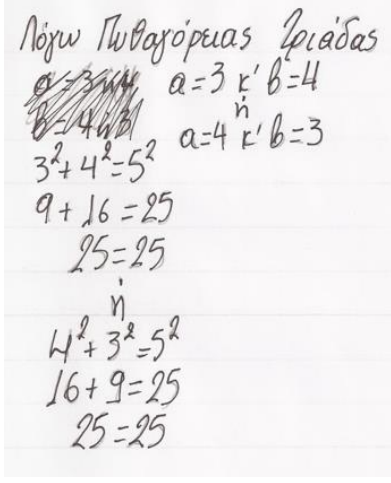

Figure 6b. $\mathrm{P}[37]$ 's response to Q1, Part II. Translation of the top line: "Because of the Pythagorean triad".

Deductive Transformational and Empirical Perceptual proof schemes (D.T. - E.P.)

A characteristic example in this class of responses is P[68]'s response to Q5 (Figure 7). P[68] 
compares sides and angles of the two triangles, $\mathrm{AB} \Gamma$ and $\mathrm{AB} \Delta$, by using the congruence criteria. Each step is justified on the grounds of known properties or given information. First, $\mathrm{A} \Gamma=\mathrm{A} \Delta$ due to the property of the perpendicular bisector of a line segment: each point of the perpendicular bisector of a segment is equidistant from the segment's endpoints and, then, $\mathrm{AB}$ is a side shared by the two triangles. The next comparison could have been the third pair of sides, $\Gamma \mathrm{B}$ and $\Delta \mathrm{B}$, and the perpendicular bisector property could have been drawn on again. $\mathrm{P}[68]$ however opts for a tougher option: to compare angles $\mathrm{BA} \Delta=\mathrm{BA} \Gamma$. These are formed by two pairs of sides that have been shown as equal $(\mathrm{AB}=\mathrm{AB}$ and $\mathrm{A} \Gamma=\mathrm{A} \Delta)$. But $\mathrm{P}[68]$ does not infer the angle congruency from this information and the theory that would allow her to do so. Instead, she concludes that "the angles are equal" by "observing the figure with the squares in the background". Our interpretation is that "the squares" in this case refers to the printed squares of the grid paper on which the given figure was sketched (see Q5 in Table 2). Finally, she concludes that the two triangles are equal due to the SAS (Side-Angle-Side) criterion. We saw her proof as logically organised overall and characterised it as D.T.. To factor in the student's justification of the angle congruency on the basis of how the angles looked like on the grid paper, we added the E.P. characterisation. Combined with the earlier characterisation of D.T., P[68]'s response to Q5 was therefore characterised as D.T. - E.P..

\begin{tabular}{|c|c|}
\hline 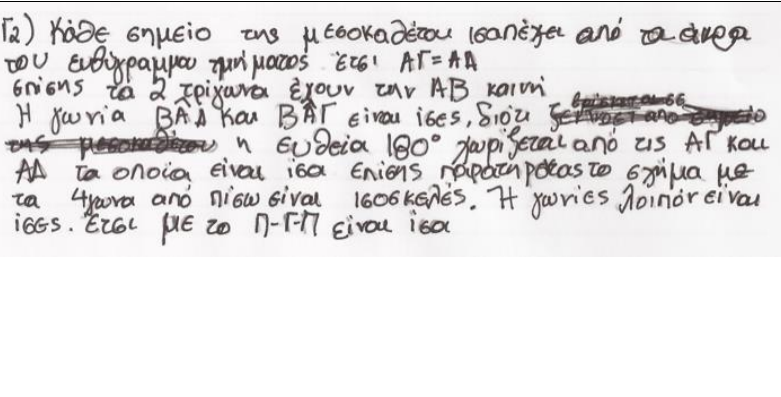 & $\begin{array}{l}\text { Every point of the perpendicular bisector is } \\
\text { equidistant from the endpoints of the line segment. } \\
\text { Thus } \mathrm{A} \Gamma=\mathrm{A} \Delta \text {. Also the } 2 \text { triangles have } \mathrm{AB} \text { in } \\
\text { common. The angle [sic] BÂA and } \mathrm{BA} \Gamma \text { are } \\
\text { equal, because [six words struck out] the line } 180^{\circ} \\
\text { is divided by } \mathrm{A} \Gamma \text { and } \mathrm{A} \Delta \text { which are equal. Also } \\
\text { by observing the figure with the [printed squares] } \\
\text { in the background is isosceles. Thus the angles are } \\
\text { congruent. As a result because of SAS [the } \\
\text { triangles] are equal. }\end{array}$ \\
\hline
\end{tabular}

Figure 7a. P[68]'s response to Q5. Figure 7b. P[68]'s translated response to Q5. 
Deductive Transformational and External Conviction Authoritarian proof schemes (D.T. EC.A.)

For this combination of proof schemes, we draw on P[65]'s response to Q3 (Figure 8). P[65] writes for Q3a that trying specific numbers is "one way" to prove that $(\boldsymbol{a}+\boldsymbol{b})(\boldsymbol{a}-\boldsymbol{b})=\boldsymbol{a}^{2}-$ $\boldsymbol{b}^{2}$ but he "would not have chosen this way". He suggests a coherent and precise algebraic proof instead. This led to the characterisation of his proof as D.T. However, because of his "this is one way to prove this" (for trying values for $\alpha$ and $\beta$ ) we were inclined to add E.I. to the characterisation. So, P[65]'s response to Q3a was characterised as D.T. - E.I..

In his response to $\mathrm{Q} 3 \mathrm{~b}$, however, $\mathrm{P}[65$ ] writes that "the teacher would want [the students] to do what I said above" (namely the deductive proof) because "this is the official proof". In this second part of the question, the student's warrant for endorsing the deductive proof is its authoritative status (“official") endorsed also by the teacher. Given the phrasing of the question, $\mathrm{P}[65]$ 's reference to the teacher is not unexpected; we see, however, the characterisation of deductive proof as acceptable because it is "official" as even stronger evidence of allusion to authority. The co-habitation in $\mathrm{P}[65]$ 's response to $\mathrm{Q} 3 \mathrm{~b}$ of appreciation for deductive proof with this allusion to authority led us to characterising this response to Q3b as D.T. - EC.A..

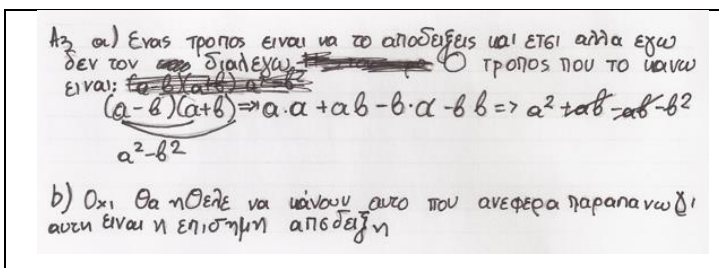

Figure 8a. P[65]'s response to Q3a,b. a) This is one way to prove it but I would have not chosen it [this way]. The way I do it is: $\frac{(a-b)(a+b)}{a^{2}-b^{2}} \Rightarrow a \cdot a+a b-b \cdot a-b b \Rightarrow a^{2}+a b=-a b-b^{2}$

b) No he [the teacher] would want [the students] to do what I said above beca[use] this is the official proof

Deductive Transformational and External Conviction Non-Referential Symbolic proof schemes (D.T. - EC.NRS.)

Here, we discuss P[85]'s response to Q2a (Figure 9). P[85] translates the data of the problem presented in the question in words (Table 1) into the correct equation. She then works on the algebraic expressions as we would expect in a deductive proof and writes $(\kappa-\lambda)(\kappa+\lambda)=\kappa+\lambda$. 
Then, she adds that "because $\kappa-\lambda=1 \kappa+\lambda=\kappa+\lambda$ " before reaching the conclusion that $\kappa-\lambda=1$.

In this response we can see evidence of a deductive argument which starts from the given information of the problem and follows logical steps towards the conclusion. For this reason, we characterised the response as D.T.. However, she uses the conclusion that she has been asked to reach ( $\kappa-\lambda=1)$ to justify the truth of this conclusion ("because"). For its lack of logical coherence, we added EC.NRS. to the D.T. characterisation of this response.

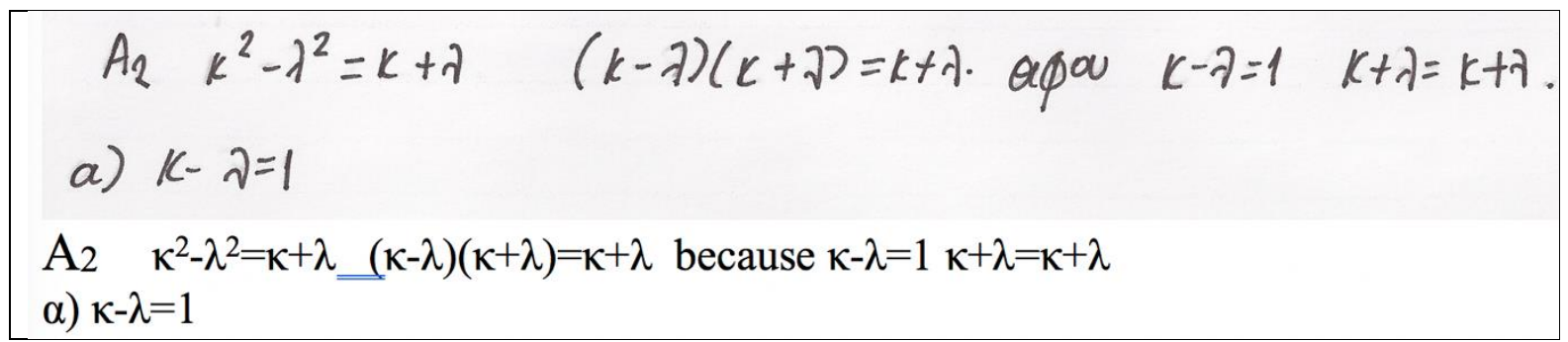

Figure 9. $\mathrm{P}[85]$ 's response to Q2a, also with translation.

Empirical Inductive and External Conviction Non-Referential Symbolic proof schemes (E.I.EC.NRS.)

For this combination, we present $\mathrm{P}[52]$ 's response to $\mathrm{Q} 1$ (Figure 10). $\mathrm{P}[52]$ substitutes $\alpha$ and $\beta$ with specific values in the algebraic expression of the problem and for this reason we characterised this response as E.I.. However, the manipulation of the numbers and the symbols has no coherent system of reference: $(\alpha \pm \beta)^{2}$ seems to become $\alpha^{2} \pm \beta^{2} ; \alpha^{2}$ and $\beta^{2}$ become $5^{2}$; and the calculations at the bottom line are not accurate. For this reason, this response was also characterised as EC.NRS.. Combined, P[52]'s response to Q1 was characterised E.I.-EC.NRS.. We note that we were tempted to characterise this response as solely EC.NRS., given the severe threat to the coherence of the script resulting from the aforementioned writing. However, we felt that the student's attempt to trial specific numbers had to somehow be acknowledged and therefore we opted for the combined characterisation E.I.-EC.NRS.. 


$$
\begin{aligned}
& \text { A1. } a, b \text { i6xua } 5^{2}=\alpha^{2}+b^{2} \\
& (a \sqrt{3}+b \sqrt{2})^{2}+(a \sqrt{2}-b \sqrt{3})^{2}=125 \\
& \left(5^{2} \cdot 9+5^{2} \cdot 4\right)+\left(5^{2} \cdot 4-5^{2} \cdot 9\right)=125 \\
& 25 \cdot 9+25 \cdot 4+25 \cdot 4-25 \cdot 9 \\
& 125+100+125-100
\end{aligned}
$$

Figure 10. $\mathrm{P}[52]$ 's response to $\mathrm{Q} 1$. The first line reads “ $\alpha, \beta$ given $5^{2}=\alpha^{2}+\beta^{2 \text { ”. }}$

\section{Empirical Perceptual and External Conviction Authoritarian proof schemes (E.P.-EC.A)}

For this combination, we present $\mathrm{P}[51]$ 's response to Q5 (Figure 11). For P[51], the two triangles, $\mathrm{AB} \Gamma$ and $\mathrm{AB} \Delta$, are congruent because they have "equal" and "same" sides and because of the "perpendicular bisector" which "separates two congruent triangles". This response indicates some justification effort: the sides of the triangles are compared and some association to the perpendicular has been attempted. However, we cannot see which congruence criterion is used and which exact sides have been compared. Additionally, the property of the perpendicular bisector as a line that "separates two congruent triangles" indicates perceptual characteristics of the perpendicular bisector that are mentioned by the student casually. We discern some coherence in this effort and, for this reason, we were reluctant to characterise the response as EC.NRS.. Furthermore, we noted that the response relies on the figure: in fact $\mathrm{P}[51]$ has reproduced the figure before responding to the question (Fig. 10a). For this reason, we characterised this response as E.P.. We also noted that the response closes with a reference to mathematical theory ("so says the theory") that supports $\mathrm{P}[51]$ 's argument about the (not-spelled out) properties of the perpendicular bisector. This wording might indicate an effort from $\mathrm{P}[51]$ to allude to a property which had been established previously in the lessons or the textbook but the student could only recall vaguely. We note that the student feels the need to warrant her claim with reference to the authority of mathematical theory. For this reason we see this reference as evidence of EC.A.. We note that we also see the student's need to warrant her claim also as evidence of willingness to participate 
in the mathematical ritual of justifying one's own claims - evidence of EC.R. - but we opt for EC.A. because of the "so says the theory" phrasing [our underlining]. Combined with the earlier characterisation of E.P., we concluded with an overall characterisation of P[51]'s response to Q5 as E.P.-EC.A..

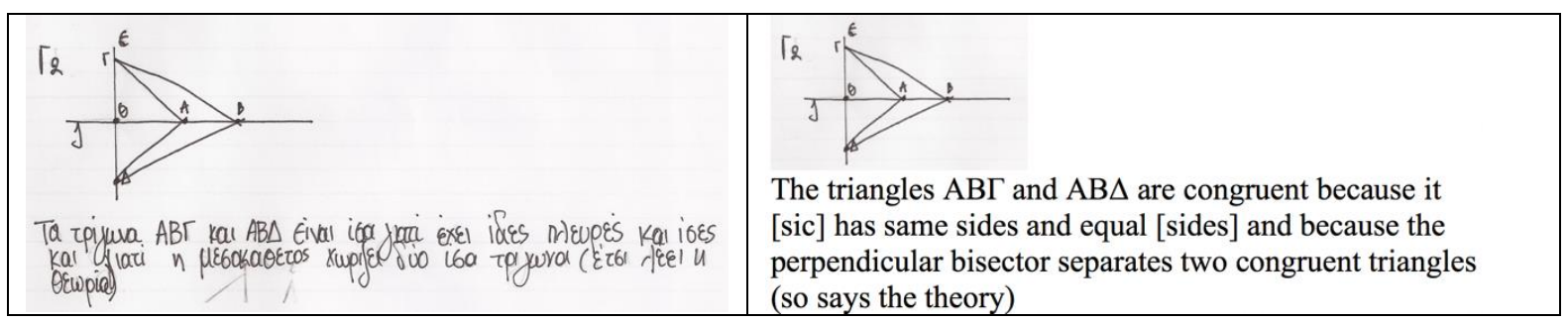

Figure 11a. P[51]'s response to Q5.

Figure 11b. P[51]'s translated response to Q5.

Empirical Perceptual and External Conviction Non-Referential Symbolic proof schemes (E.P.EC.NRS.)

For this combination, we present $\mathrm{P}[11]$ 's response to $\mathrm{Q} 4 \mathrm{~b}$ (Figure 12). $\mathrm{P}[11]$ does not sketch perpendicular segments from vertices $A$ and $\Gamma$ as the problem indicated. The segments in her figure seem to us to connect the vertices with the mid-points of the opposite sides of the parallelogram (but we note the speculative character of this interpretation). In her text, she identifies $\mathrm{A} \Delta$ and $\mathrm{B} \Gamma$ as parallel and equal "since this is a parallelogram". Because of the way she drew the figure, $\mathrm{AE}$ and $\mathrm{Z} \Gamma$ look parallel and equal (the actual $\mathrm{AE}$ and $\mathrm{Z} \Gamma$ requested in the question are also parallel and equal as they are line segments that are perpendicular to parallel lines). For this reason, we characterised her response as E.P.. Furthermore, possibly emanating from her (correct but not spelled out) recall of a parallelogram's properties, she writes that $\hat{\Delta}=\hat{\mathrm{B}}$ and $\hat{\Gamma}=\hat{\mathrm{A}}$. So, in her view, she has established that the two triangles $\mathrm{A} \Delta \mathrm{E}$ and $\mathrm{B} \Gamma \mathrm{Z}$ are equal because of an (also not spelled out) congruence criterion she was taught in the lessons. Given the lack of coherence in the flow of her argument, we characterised this response also as EC.NRS.. Combined with the E.P. characterisation, this gives an overall characterisation of $\mathrm{P}[11]$ 's response to Q4b as E.P.-EC.NRS.. 


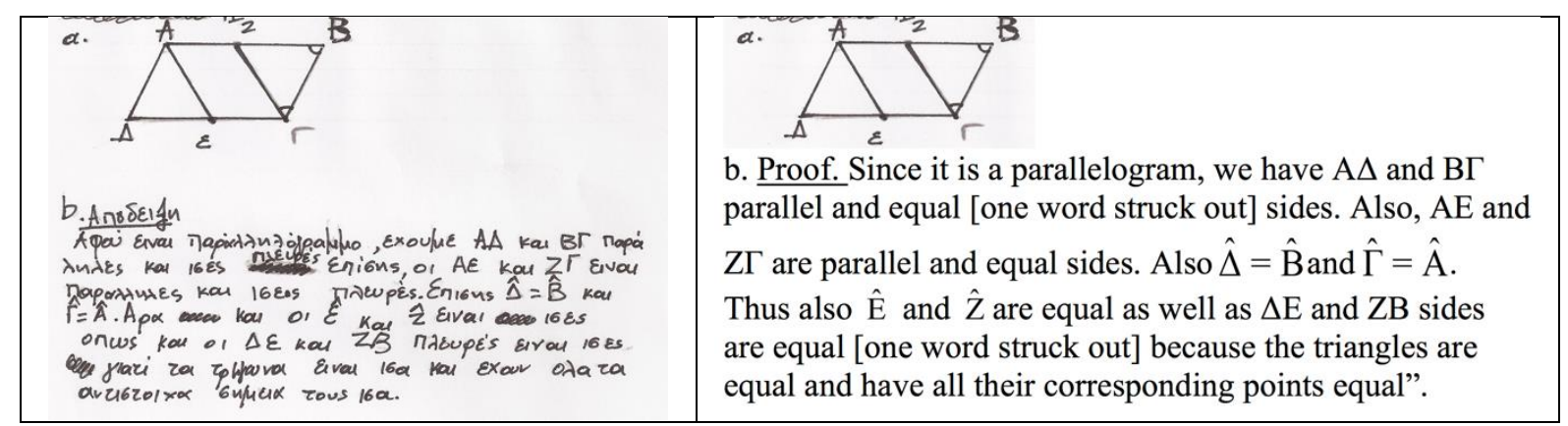

Figure 12a. $\mathrm{P}[11]$ 's response to $\mathrm{Q} 4 \mathrm{~b}$. Figure 12b: $\mathrm{P}[11]$ 's translated response to $\mathrm{Q} 4 \mathrm{~b}$.

External Conviction Ritual and External Conviction Non-Referential Symbolic proof schemes (EC.R.-EC.NRS.)

For the last proof scheme combination we exemplify in this paper, we present $\mathrm{P}[32]$ 's response to Q4b (Figure 13). P[32] constructs the correct figure. Then, she organises her response by writing the "Hypothesis", the "Conclusion" and, finally, the "Proof". In the proof, she names the triangles that she compares, and, underneath, she lists the equal sides by adding the explanation in brackets, in this case "by definition". She completes the proof with the congruency criterion she uses, most likely the SSS (Side-Side-Side) criterion according to the Year 9 textbook (Argyrakis, et al., 2010), and the conclusion that the triangles are congruent. This style of response was very common in the lessons and in the textbook (see also earlier comment on EC.R. proof scheme evidence in the scripts). This is the presentation style the teacher expects from the students and $\mathrm{P}[32]$ followed suit. Thus, we characterised this response as EC.R.. However, P[32]'s response lacks coherence. Two examples of this incoherence are: (1) one of two pairs of sides that she claims are equal, $\mathrm{AB}$ and $\Delta \Gamma$, do not belong to the triangles she compares and (2) the claim that because "the figure is a tilted parallelogram" then "all its sides are equal" is not valid and does not justify the derivation of $\mathrm{AE}=\mathrm{Z} \Gamma$ and $\mathrm{AE} / / \mathrm{Z} \Gamma$. This led to an EC.NRS. characterisation. Together with the earlier characterisation of EC.R.., we concluded with an overall characterisation of P[32]'s response to Q4b as EC.R.-EC.NRS.. 


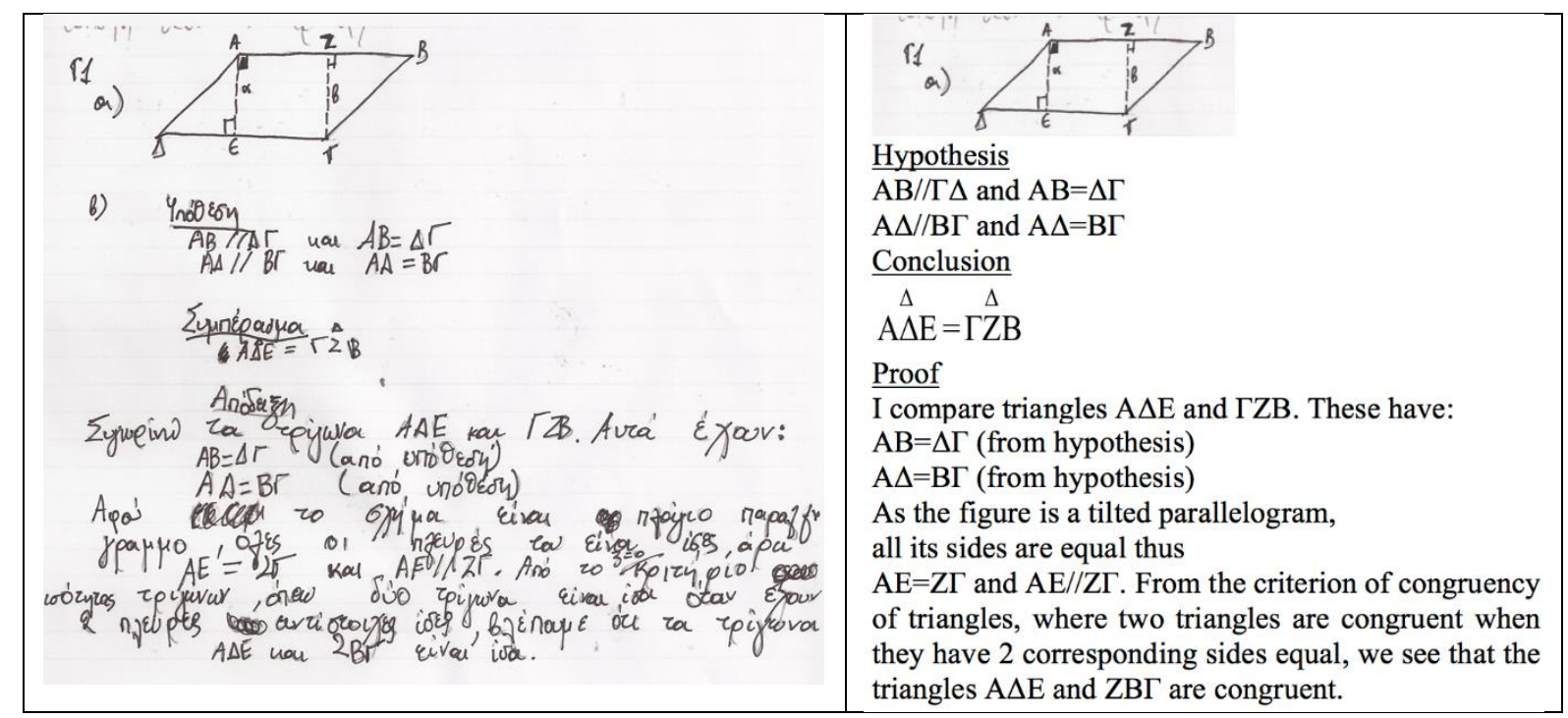

Figure 13a. $\mathrm{P}[32]$ 's response to $\mathrm{Q} 4 \mathrm{~b}$.

Figure 13b. P[32]'s translated response to Q4b.

\section{The theoretical and pedagogical potency of the enriched and extended application of}

\section{Harel and Sowder's proof schemes taxonomy}

The study (Kanellos, 2014) on which we draw in this paper investigates secondary students' emergent learning of proof and proving in relation to the ultimate goal of deductive proof production, stated as a statutory requirement in the national curriculum - and respective textbook (Argyrakis, et al., 2010) - of the country (Greece) in which the study took place. The study adopted the analytical lenses of the Harel and Sowder (2007) proof schemes taxonomy and investigated the question: can the Harel and Sowder proof schemes taxonomy be deployed in the characterization of secondary students' first encounter with proof and proving? If so, how? We analysed data from 85 students from four Year 9 (age 14 to 15) classes studying in a mixed ability, state-funded, Greek secondary school. According to the Greek curriculum, Year 9 students begin to practise proof examples in algebra and geometry. Although introduction to the axiomatic foundations (e.g. Euclidean Geometry) and to proving in the context of these foundations come later in their studies (Year 10), we have considered Year 9 as a transition point in students' first introduction to proof from empirical verification of mathematical statements to logical reasoning and structured argumentation based on definitions and properties of mathematical objects. The data from which we report in this paper are students' responses to a questionnaire distributed close to the end of the school year and included questions from both algebra and geometry.

The analysis identified evidence of six of the seven proof schemes proposed by Harel 
and Sowder (2007): external conviction (ritual, authoritarian, non-referential symbolic); empirical (inductive, perceptual); and, deductive transformational proof schemes. As we expected, we did not identify evidence of the deductive axiomatic proof scheme in the student responses. This is not surprising given that the students have not been introduced to mathematics in terms of its axiomatic foundations. Additionally, our analysis revealed high occurrence of responses in which we saw evidence of more than one proof scheme. In resonance with previous studies, such as Housman and Porter (2003), which have identified evidence of different proof schemes in responses offered by the same student, we engaged with characterisations of students' responses that encompassed more than one proof scheme. In characterising the concurrent evidencing of more than one proof scheme in the same response, we identified eight combinations of the above six proof schemes (Table 3). In this paper we exemplified each of the eight combinations with characteristic examples of student responses.

In the first stages of our analysis, we wondered about the order of the proof schemes in the combined characterisations. For example, is D.T.-EC.NRS. different from EC.NRS.-D.T.? As the analysis progressed, we realised that the order does not matter. We agreed to combine all the proof schemes for which our team could see enough evidence of and we did so through several cycles of triangulating discussion of all cases we saw as ambiguous. Throughout this process, we realised that we prioritised a proof scheme that we saw as more salient, while at the same time identifying evidence of other proof schemes. We stress that, in our analysis, a student response in which evidence of more than one proof scheme can be identified has significant pedagogical interest: a teacher alerted to this concurrent evidencing of more than one proof scheme in the same response, can pick up the potential ambiguity that this may imply and investigate further, discuss the implications explicitly and orchestrate classroom activity in a way that facilitates the endorsement by the class of, for example, deductively generated arguments. 
Furthermore, we noticed that there was a small number of responses with evidence of more than two proof schemes. We opted to keep the two most salient in our characterisation. We conjecture though that a different data collection design that allows more insight in students' proving activity (e.g. through task-based interviews where participants may also be invited to think aloud while engaged with the tasks) may reveal evidence of a range of proof schemes in the proving activity of the same individual. This remains to be investigated.

In this paper, we focus on illustrating the proof scheme combinations we identified in the student responses and to indicate the type of nuanced insight into the students' emerging familiarisation with proof and proving that this analysis allows. Here, we do not discuss potential reasons that may facilitate the emergence of certain proof schemes against others as reporting the results of our analysis of the 85 students' responses is not the main aim of this paper. However, we feel compelled to mention a significant observation, elaborated in Kanellos (2014): although the tasks were familiar but relatively demanding for the students, students' performance was overall to a good standard. Indicatively, 74\% of the students responded to Q5 and $45 \%$ of the students gave a response that was characterised as D.T. or D.T. combined with other proof schemes. This is encouraging evidence in favour of the claim that students as early in their secondary school education as Year 9, are notably ready for an introduction to proof. This is naturally subject to appropriate curricular design and instruction. As Kanellos (2014) notes, credit with regard to the latter (instruction) must go to the teacher who taught the - by all means unexceptional, mixed ability, indeed typical - classes that participated in the study.

Overall, our analysis resonates with Housman and Porter's (2003) finding that the same student may follow different proof schemes in different tasks. It seems that certain types of task may facilitate the emergence of certain types of proof behaviour. For example, most of the evidence on E.I. (empirical inductive) proof schemes was identified in the responses to the algebra questions, whereas most of the evidence on E.P. (empirical perceptual) proof schemes 
was identified in the responses to the geometry questions. Q3 was included in the questionnaire to give more insight on whether students appreciate a deductive response and what they believe an acceptable proof is to their teacher (inspired by Healy \& Hoyles, 2000). This question gave us evidence of the EC.A. (external conviction authoritarian) proof scheme that was not apparent in the responses to other questions.

The proof scheme taxonomy emerged initially in a post-secondary context (Harel and Sowder, 1998) and we were apprehensive about the appropriateness of our choice to use these theoretical lenses on data from the secondary level and for an investigation of students' first encounters with proof. Nevertheless, we were interested in studying student proof activity in relation to deductive proof - in which the curriculum and their teacher are aiming to enculturate the students - and this taxonomy has the potential to interpret this activity. Our analysis provides strong evidence that an enriched and extended application of Harel and Sowder's proof schemes taxonomy with combined proof schemes can indeed describe the multifaceted and evolving student responses. We stress that we see the proof scheme combinations as a dynamic tool - not a static taxonomy - and we conjecture that further combinations may still emerge from analyses of other datasets.

Our outcomes go beyond previous research on secondary school student proof activity that report different proof schemes adopted by different participants in the same task or the existence of different proof schemes in the responses to different questions by the same student (Housman \& Porter, 2003). Also, our study substantiates observations of different tendencies appearing simultaneously in the response to the same question by the same participant (Lee, 2016) and offers a tool to describe these observations.

While the study is embedded in the particular secondary education context of one country (Greece), we see the analytical framework that we propose in this paper - the application of sole and combined proof schemes taxonomy - as likely to be appropriate for 
analogous datasets collected in other educational contexts. As we can see in the overview of characterisation from the sample of the participants we discuss in this paper (Table 4), there is a variation of characterisations for the same participant across tasks, for the same task across participants and internally within a specific response. We propose that this use of the sole and combined proof schemes has the theoretical potency to portray emerging elements of deductive proof behaviour, such as generality, operational thought and logical inference, in the midst of empirical and external conviction elements which are still clearly present in students' proving activity. Through this type of analysis, we can trace the emerging preparedness of the students for proof and proving while we can evidence the reasoning habits that hold them back. Furthermore, we see the pedagogical potency of the proposed proof scheme combinations as teachers can deploy the enriched taxonomy towards characterising their students' multi-faceted and evolving proving activity and thus inform their decision-making on how to best facilitate their students' enculturation in formal mathematical reasoning. As Nardi and Knuth (2017) note, "orchestrating a focus on proof appreciation requires a change in classroom culture with respect to proof and proving, a change that must start with teachers" (p. 269). We see the use of the sole and combined proof schemes proposed in this paper as being of assistance to teachers and researchers interested in noticing the signals of where this change of classroom culture may be on the cusp of having the capacity to occur.

We credit the enriched and extended application of Harel and Sowder's proof schemes taxonomy that we devised and deployed in this study for revealing these students' learning trajectories with remarkable nuance. We see the use of the sole and combined proof schemes as a potent diagnostic tool that can bring research and teaching of proof and proving a step closer to achieving a worthy goal: tapping effectively into young adults' potential for a fuller appreciation of - and competence in - a quintessential aspect of mathematics, proof and proving. 


\section{Acknowledgements}

We thank the teacher and students who participated in the study very warmly.

\section{References}

Argyrakis, D., Vourganas, P., Mentis, K., Tsikopoulou, S., \& Chryssovergis, M. (2010).

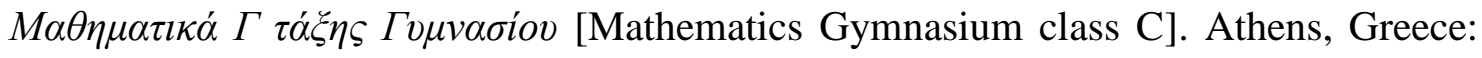
OEDB.

Balacheff, N. (1987). Processus de preuve et situations de validation [Proving Processes and Situations for Validation]. Educational Studies in Mathematics, 18(2), 147-176.

Biza, I., Nardi, E., \& Zachariades, T. (2009). Teacher beliefs and the didactic contract on visualisation. For the Learning of Mathematics, 29(3), 31-36.

Csíkos, C. A. (1999). Measuring students' proving ability by means of Harel and Sowder's proof- categorization. In O. Zaslavsky (Ed.), Proceedings of the 23rd Conference of the International Group for the Psychology of Mathematics Education (Vol. 2, pp. 233-240). Haifa, Israel: PME.

Hanna, G. (1991). Mathematical proof. In D. Tall (Ed.), Advanced Mathematical Thinking (pp. 54-61). Dordrecht, The Netherlands: Kluwer Academic Publishers.

Hanna, G. (2000). Proof, explanation and exploration: An overview. Educational Studies in Mathematics, 44(1-3), 5-23.

Hanna, G. (2007). The ongoing value of proof. In P. Boero (Ed.), Theorems in School: From History, Epistemology, and Cognition to Classroom Practice, (pp. 3-16). Rotterdam, The Netherlands: Sense Publishers.

Hanna, G., \& Barbeau, E. (2008). Proofs as bearers of mathematical knowledge. ZDM The International Journal on Mathematics Education, 40(3), 345-353.

Harel, G. (1999). Students' understanding of proofs: A historical analysis and implications for the teaching of geometry and linear algebra. Linear Algebra and its Applications, 302- 
$303,601-613$.

Harel, G. (2007). Students' proof schemes revisited. In P. Boero (Ed.), Theorems in School: From History, Epistemology, and Cognition to Classroom Practice, (pp. 65-78). Rotterdam, The Netherlands: Sense Publishers.

Harel, G., \& Sowder, L. (1998). Students' proof schemes: Results from exploratory studies. In A. Schoenfeld, J. Kaput, \& E. Dubinsky (Eds.), Research in collegiate mathematics education III (pp. 234-283). Providence, RI: American Mathematical Society.

Harel, G., \& Sowder, L. (2007). Toward a comprehensive perspective on proof. In F. Lester (Ed.), Second Handbook of Research on Mathematics Teaching and Learning, (pp. 805842). National Council of Teachers of Mathematics.

Healy, L., \& Hoyles, C. (2000). A Study of Proof Conceptions in Algebra. Journal for Research in Mathematics Education, 31(4), 396-428.

Heinze, A. (2004). Schülerprobleme beim Lösen von geometrischen Beweisaufgaben-eine Interviewstudie. ZDM The International Journal on Mathematics Education, 36(5), 150161.

Housman, D., \& Porter, M. (2003). Proof schemes and learning strategies of above-average mathematics students. Educational Studies in Mathematics, 53(2), 139-158.

Kanellos, I. (2014). Secondary students' proof schemes during the first encounters with formal mathematical reasoning: appreciation, fluency and readiness. Unpublished doctoral thesis. University of East Anglia: UK. Available at: https://ueaeprints.uea.ac.uk/49759/1/2014KanellosIEdD.pdf

Kanellos, I., Nardi, E., \& Biza, I. (2013). The interplay between fluency and appreciation in secondary students' first encounter with proof. In A.M. Lindmeier \& A. Heinze (Eds.), Proceedings of the 37th Conference of the International Group for the Psychology of Mathematics Education (Vol. 5, pp. 84). Kiel, Germany: PME. 
Küchemann, D., \& Hoyles, C. (2006). Influences on students' mathematical reasoning and patterns in its development: Insights from a longitudinal study with particular reference to geometry. International Journal of Science and Mathematics Education, 4(4), 581608.

Lee, K. (2016). Students' proof schemes for mathematical proving and disproving of propositions. Journal of Mathematical Behavior, 41, 26-44.

Nardi, E., \& Knuth, E. (2017). Changing classroom culture, curricula, and instruction for proof and proving: How amenable to scaling up, practicable for curricular integration, and capable of producing long-lasting effects are current interventions? Educational Studies in Mathematics, 96(2), 267-274.

Skemp, R. R. (1976). Relational understanding and instrumental understanding. Mathematics Teaching, 77, 20-26.

Stylianides, A. J. (2007). The notion of proof in the context of elementary school mathematics. Educational Studies in Mathematics, 65(1), 1-20.

Stylianides, A. J., Stylianides, G. J., \& Philippou, G. N. (2004). Undergraduate Students' Understanding of the Contraposition Equivalence Rule in Symbolic and Verbal Contexts. Educational Studies in Mathematics, 55(1/3), 133-162.

Stylianides, G. J., \& Stylianides, A. J. (2008). Proof in School Mathematics: Insights from Psychological Research into Students' Ability for Deductive Reasoning. Mathematical Thinking and Learning, 10(2), 103-133.

Weber, K., \& Alcock, L. (2004). Semantic and Syntactic Proof Productions. Educational Studies in Mathematics, 56(2), 209-234. 